\title{
Bariatric Surgery Outcomes in Patients with Prior Solid Organ Transplantation: an MBSAQIP Analysis
}

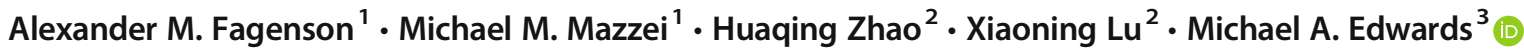 \\ Published online: 24 February 2020 \\ (C) Springer Science+Business Media, LLC, part of Springer Nature 2020
}

\begin{abstract}
Introduction Obesity is a risk factor for poor patient outcomes after organ transplantation (TXP). While metabolic and bariatric surgery (MBS) is safe and effective in treating severe obesity, the role of MBS in transplant patients continues to evolve.

Methods A retrospective analysis was performed of sleeve gastrectomy (SG) and Roux-en-Y gastric bypass (RYGB) patients in the 2017 Metabolic and Bariatric Surgery Accreditation Quality and Improvement Project (MBSAQIP) database. Propensity and case-control matching, and multivariable logistic regression were performed for 30-day post-operative outcomes.

Results A total of 336 transplant patients were compared with 157,413 patients without transplant. Propensity and case-control matching reveal no significant differences in mortality $(p>0.2)$. However, case-control matching revealed longer operative time (104 min versus $76 \mathrm{~min}, p<0.001$ ), increased length of stay ( 2 days versus 1 day, $p<0.05$ ), perioperative transfusions ( $2 \%$ versus $0.22 \%, p=0.009)$, and leak rates $(2.2 \%$ versus $0.55 \%, p=0.02)$ in the transplant cohort. On multivariable regression analysis, prior transplantation was associated with higher rates of overall $(\mathrm{OR} 1.6, p=0.007)$ and bariatric-related morbidity $(\mathrm{OR} 1.78, p=$ $0.004)$, leak (OR 3.47, $p=0.0027)$, and surgical site infection (OR 3.32, $p=0.004)$. Prior transplantation did not predict overall $(p=0.55)$ nor bariatric-related mortality $(p=0.99)$.

Conclusion MBS in prior solid organ transplantation patients is overall safe, but is associated with increased operative time and length of stay, as well as higher rates of some post-operative morbidity.
\end{abstract}

Keywords Bariatric surgery $\cdot$ Solid organ transplantation $\cdot$ MBSAQIP $\cdot$ Perioperative outcomes

\section{Introduction}

Obesity is increasingly prevalent after solid organ transplantation, and may negatively impact the transplant population on multiple levels [1-6]. Obesity in transplantation patients may also negatively impact perioperative and long-term outcomes after metabolic and bariatric surgery (MBS) [7-11]. In the systematic review by Sood et al., obesity was associated with a higher odds ratio for biopsy-proven acute rejection, mortality, allograft loss, and the development of diabetes [8].

Michael A. Edwards

Edwards.michael@mayo.edu; maeedw@gmail.com

1 Department of Surgery, Temple University Hospital, Suite, 3401 North Broad Street, Philadelphia, PA 19140, USA

2 Department of Clinical Sciences, Lewis Katz School of Medicine at Temple University, Philadelphia, PA 19140, USA

3 Department of Surgery, Mayo Clinic, 4500 San Pablo Rd S, Jacksonville, FL 32224, USA
Patients with a history of solid organ transplantation are routinely considered to be high-risk patients. This risk stratification is compounded by the presence and disease burden of obesity. Given its safety profile and health impact, there is increasing interest in the role of MBS in obese patients with prior organ transplantation. Therefore, the aim of this study is to compare outcomes of the largest North American patient cohort, with and without a history of solid organ transplantation, undergoing metabolic and bariatric surgery.

\section{Material and Methods}

\section{Data Source}

We performed a retrospective analysis of data from the 2017 Metabolic and Bariatric Surgery Accreditation and Quality Improvement Program (MBSAQIP) Participant Use Files (PUF) database, and compared outcomes between those with and without a history of previous solid organ transplantation. 
The MBSAQIP is responsible for the accreditation of bariatric surgical facilities. Requirements for certification include reporting bariatric surgical outcomes to the MBSAQIP Participant Use Data File (PUF), a Health Insurance Portability and Accountability Act (HIPAA)-compliant data file registry containing prospectively entered, risk-adjusted, clinically rich data using standardized definitions for preoperative, intraoperative, and post-operative variables that are specific to metabolic and bariatric surgical care. Data points are abstracted at participating institutions by certified reviewers who are audited for accuracy of performance. For the first time, the 2017 file included data on previous solid organ transplantation, including a history of heart, lung, liver, renal, pancreas, and bowel transplantation. The database does not give the ability to discern which type of transplant has been performed. This is a de-identified, nationally available, clinical database; therefore, neither institutional review board (IRB) approval nor patient consent was required for our study.

\section{Case Selection and Inclusion Criteria}

A patient selection diagram is shown in Fig. 1. Participants included patients who had a primary gastric bypass (RYGB) or sleeve gastrectomy (SG) in 2017, designated by Current Procedural Terminology (CPT) codes 43644, 43645, and 43775. We excluded patients less than 18 years or greater than 80 years old, body mass index $(\mathrm{BMI})<35$, any bariatric procedure other than a RYGB or SG, bariatric procedures designated as emergency, open cases, revision/conversion cases, and those with incomplete clinical data. Selected cases were further stratified by a history of solid organ transplantation (TXP). There were 614 TXP patients in the 2017 MBSAQIP database prior to exclusions. 336 were included in our analysis. A total of 278 TXP patients were excluded from analysis

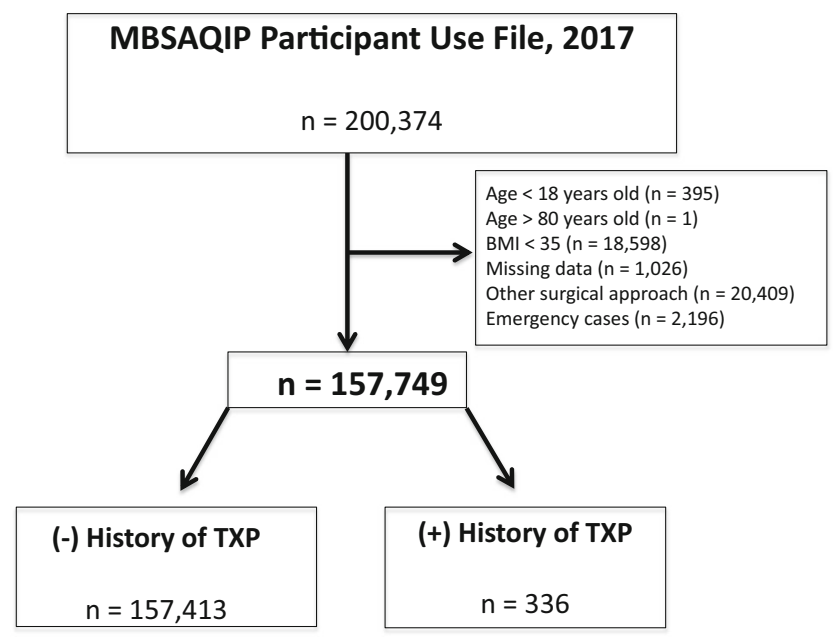

Fig. 1 Patient selection strategy for the following reasons: age $<18$ or $>80$ years old $(n=1)$, BMI $<35(n=136)$, having a prior bariatric surgery $(n=40)$, emergency cases $(n=22)$, open surgical approach $(n=9)$, and incomplete data $(n=70)$.

\section{Data Collection and Statistical Analysis}

Descriptive statistics were collected and compared between groups, including demographics, health summary status, preoperative comorbidities, and operative characteristics. Primary outcome measures included 30-day mortality and morbidity. Secondary outcome measures included other 30-day adverse outcomes (reoperation, readmission, and reintervention), post-operative complication, composite complications, operative duration, conversion, and hospital length of stay. Unmatched cohorts were compared by univariate analysis, using Pearson chi-square test for categorical variables and Mann-Whitney $U$ test for continuous variables.

A backward method multivariable logistic regression was performed based on those preoperative variables (demographics, health status, comorbidities) that were statistically significant $(p<0.05)$ between cohorts in unmatched analysis. Variables in our regression methodology included, age, BMI, gender, race, American society of anesthesia (ASA) class, operation type, history of myocardial infarction, percutaneous coronary intervention, cardiac surgery, hypertension, hyperlipidemia, diabetes mellitus, smoking, renal insufficiency, dialysis, deep venous thrombosis requiring therapy, pulmonary embolism, inferior vena cava filter, and anticoagulation for presumed or confirmed venous thromboembolism (VTE) and chronic steroids.

\section{Matching}

Propensity and case-control matched analyses were performed to account for inter-group biases. For both propensity score and case-control matching, the ratio of transplant recipients to control patients without transplantation was 1:5. For propensity score matching, a logistic regression model was generated on variables significantly different $(p<0.05)$ on univariate analysis between those with and without a history of solid organ transplantation. Matching variables included age, sex, race, BMI, operation type, diabetes mellitus, hypertension, hyperlipidemia, ASA class, steroid use, renal insufficiency, dialysis status, smoking status, history of pulmonary embolism, history of IVC filter preoperatively, history of VTE requiring therapy, and anticoagulation use preoperatively. A propensity score from 0 to 1 was generated from this model and assigned to each subject. A nearest-neighbor variable ratio with propensity scores that fell within a caliper of 0.05 was then used to generate matched cohorts hypothesized to be balanced on potentially confounding baseline characteristics. 
For case-control matched analysis, cases and controls were matched based on clinical variables that were significantly different in univariate analysis of the unmatched cohorts. This resulted in matched cohorts with equal distributions of those variables, including age, sex, race, BMI, operation type, diabetes mellitus, hypertension, hyperlipidemia, ASA class, steroid use, renal insufficiency, dialysis status, smoking status, history of pulmonary embolism, history of IVC filter preoperatively, history of VTE requiring therapy, and anticoagulation use preoperatively.

Primary and secondary outcomes were compared with Pearson chi-square test for categorical variables and MannWhitney $U$ test for continuous variables. Continuous data is expressed as median and interquartile range (IQR) and categorical data is expressed as frequency and percentage. Aggregate complications (Appendix 1 Table 6) were also compared, including aggregate leak, bleeding, renal, cardiovascular and pulmonary complications, venous thromboembolic events, aggregate surgical site infection, and other infection. All statistical analyses were performed with SPSS version 25 (IBM Corporation, Armonk, NY) and SAS version 9.4 (SAS Institute, Cary, NC). A $p$ value of $<0.05$ was considered statistically significant.

\section{Results}

\section{Demographics of Study Cohorts}

Table 1 shows the unmatched patient characteristics of the two cohorts. After exclusions, we identified 336 metabolic and
Table 1 Patient characteristics of unmatched cohorts

\begin{tabular}{|c|c|c|c|}
\hline & $\begin{array}{l}(-) \text { TXP } \\
{[n=157,413]}\end{array}$ & $\begin{array}{l}(+) \text { TXP } \\
{[n=336]}\end{array}$ & $p$ value \\
\hline \multicolumn{4}{|l|}{ Continuous variables, median (IQR) } \\
\hline Age (years) & $44(35-53)$ & $48(39-57)$ & 0.040 \\
\hline BMI closest to surgery $\left(\mathrm{kg} / \mathrm{m}^{2}\right)$ & $43.94(40.15-49.28)$ & $42.53(39.33-46.18)$ & 0.010 \\
\hline \multicolumn{4}{|l|}{ Categorical variables, $n(\%)$} \\
\hline Gender (female) & $126,002(80)$ & $232(69)$ & $<0.001$ \\
\hline Race (White) & $98,528(63)$ & $175(52)$ & $<0.001$ \\
\hline Race (Black) & $28,607(18)$ & $76(23)$ & 0.035 \\
\hline Ethnicity (Hispanic) & $19,718(13)$ & $53(16)$ & 0.073 \\
\hline ASA class & & & $<0.001$ \\
\hline$<3$ & $34,232(22)$ & $34(10)$ & \\
\hline$>3$ & $123,181(78)$ & $302(90)$ & \\
\hline Operation type & & & $<0.001$ \\
\hline Sleeve & $114,290(72)$ & $260(77)$ & \\
\hline Gastric bypass & $43,123(27)$ & $76(23)$ & \\
\hline Surgical approach & & & 0.919 \\
\hline Laparoscopic & $144,536(92)$ & $308(92)$ & \\
\hline Robotic & $12,877(8)$ & $28(8)$ & \\
\hline \multicolumn{4}{|c|}{ Preoperative disease prevalence, $n(\%)$} \\
\hline History of MI & $1870(1)$ & $8(2)$ & 0.044 \\
\hline History of PCI & $2814(2)$ & $15(4)$ & $<0.001$ \\
\hline History cardiac surgery & $1547(1)$ & $21(6)$ & $<0.001$ \\
\hline Hypertension & $74,576(47)$ & $229(68)$ & $<0.001$ \\
\hline Hyperlipidemia & $35,554(23)$ & $132(39)$ & $<0.001$ \\
\hline Diabetes mellitus & $39,710(25)$ & $131(39)$ & $<0.001$ \\
\hline COPD & $2467(2)$ & $3(1)$ & 0.320 \\
\hline OSA & $60,224(38)$ & $114(34)$ & 0.103 \\
\hline Oxygen dependent & $1117(1)$ & $4(1)$ & 0.295 \\
\hline Smoker & $13,067(8)$ & $12(4)$ & 0.002 \\
\hline Renal insufficiency & $914(1)$ & $47(14)$ & $<0.001$ \\
\hline Dialysis & $466(0.3)$ & $30(9)$ & $<0.001$ \\
\hline VTE requiring therapy & $1783(1.40)$ & $1013(1.64)$ & $<0.001$ \\
\hline History of PE & $1931(1)$ & $12(4)$ & $<0.001$ \\
\hline IVC filter & $839(1)$ & $5(2)$ & 0.017 \\
\hline Anticoagulation & $4451(3)$ & $23(7)$ & $<0.001$ \\
\hline Chronic steroids & $2755(2)$ & $144(43)$ & $<0.001$ \\
\hline Limited ambulation status & $2312(1)$ & $9(3)$ & 0.066 \\
\hline Independent functional status & $155,684(99)$ & $329(98)$ & 0.084 \\
\hline History of bariatric surgery & $10,834(7)$ & $26(8)$ & 0.536 \\
\hline
\end{tabular}

TXP history of solid organ transplantation, $I Q R$ interquartile range, $\mathrm{kg}$ kilogram, ASA American Society of Anesthesiologist, $M I$ myocardial infarction, $P C I$ percutaneous coronary intervention, $C O P D$ chronic obstructive pulmonary disease, $O S A$ obstructive sleep apnea, $P E$ pulmonary emboli, IVC inferior vena cava, VTE venous thromboembolism 
bariatric surgery cases with a history of prior solid organ transplantation and 157,413 cases without. The transplant cohort had a higher median age ( 48 years vs. 44 years, $p=0.04$ ) and a lower median BMI $\left(42.5 \mathrm{~kg} / \mathrm{m}^{2}\right.$ vs. $\left.43.9 \mathrm{~kg} / \mathrm{m}^{2}, p=0.009\right)$, and was less likely to be female $(69 \%$ vs. $80 \%, p<0.001)$. Surgical approaches were similar between cohorts. The transplant cohort had significantly $(p<0.05)$ higher rates of cardiovascular disease and cardiac risks (history of MI, PCI cardiac surgery, hypertension, hyperlipidemia, and diabetes mellitus), chronic kidney disease, and prior venous thromboembolism.
Smoking was more prevalent in the cohort without prior organ transplantation (Table 1).

\section{Outcomes Following Unmatched Cohort Analysis}

Outcomes of the unmatched cohorts are detailed in Table 2. There was no mortality difference $(p=0.17)$ between those who had previously undergone TXP and those who had not. Overall morbidity $(12 \%$ vs. $5 \%, p<0.001)$ and bariatricrelated morbidity $(9 \%$ vs. $4 \%, p<0.001)$ were both signifi-
Table 2 Outcomes, unmatched cohorts

\begin{tabular}{|c|c|c|c|}
\hline & $\begin{array}{l}(-) \text { TXP } \\
{[n=157,413]}\end{array}$ & $\begin{array}{l}(+) \text { TXP } \\
{[n=336]}\end{array}$ & $p$ value \\
\hline Operative time, $(\mathrm{min})^{*}$ & $74(53-108)$ & $99(67-136)$ & $<0.001$ \\
\hline Hospital LOS (days)* & $1(1-2)$ & $2(1-2)$ & $<0.001$ \\
\hline \multicolumn{4}{|c|}{ 30-day adverse outcomes and perioperative complications $n(\%)$} \\
\hline Mortality & $130(0.08)$ & $1(0.3)$ & 0.170 \\
\hline Death related & $77(0.05)$ & $1(0.3)$ & 0.410 \\
\hline Overall morbidity & $7660(5)$ & $40(12)$ & $<0.001$ \\
\hline Overall morbidity related & $5781(4)$ & $29(9)$ & $<0.001$ \\
\hline Reoperation & $1898(1)$ & $6(2)$ & 0.330 \\
\hline Reoperation related & $1526(1)$ & $6(2)$ & 0.130 \\
\hline Readmission & $5730(4)$ & $33(10)$ & $<0.001$ \\
\hline Readmission related & $4511(3)$ & $25(7)$ & $<0.001$ \\
\hline Post-op intervention & $1838(1)$ & $8(2)$ & 0.039 \\
\hline Post-op intervention, related & $1591(1)$ & $8(2)$ & 0.012 \\
\hline ICU admission & $1043(1)$ & $10(3)$ & $<0.001$ \\
\hline Follow-up & $149,903(95)$ & $319(95)$ & 0.800 \\
\hline Transfusion & $993(1)$ & $9(3)$ & $<0.001$ \\
\hline Acute renal failure & $97(0.06)$ & $2(0.6)$ & $<0.001$ \\
\hline Progressive renal failure & $96(0.06)$ & $4(1.19)$ & $<0.001$ \\
\hline CPR & $64(0.04)$ & $0(0)$ & 0.710 \\
\hline Stroke & $20(0.01)$ & $0(0)$ & 0.840 \\
\hline Myocardial infarction & $36(0.02)$ & $1(0.3)$ & $<0.001$ \\
\hline DVT requiring therapy & $280(0.18)$ & $1(0.3)$ & 0.600 \\
\hline Pulmonary embolism & $175(0.11)$ & $2(0.6)$ & 0.008 \\
\hline Pneumonia & $313(0.2)$ & $2(0.6)$ & 0.100 \\
\hline Reintubation & $181(0.11)$ & $0(0)$ & 0.530 \\
\hline Superficial SSI & $679(0.43)$ & $4(1.2)$ & $<0.001$ \\
\hline Deep incisional SSI & $100(0.06)$ & $1(0.3)$ & 0.090 \\
\hline Organ space SSI & $354(0.22)$ & $3(0.89)$ & 0.030 \\
\hline Post-operative sepsis & $153(0.1)$ & $0(0)$ & 0.850 \\
\hline Post-operative septic shock & $95(0.06)$ & $0(0)$ & 0.650 \\
\hline Post-operative UTI & $564(0.37)$ & $1(0.3)$ & 0.970 \\
\hline C. diff & $188(0.12)$ & $1(0.3)$ & 0.350 \\
\hline Incisional hernia & $109(0.07)$ & $1(0.3)$ & 0.110 \\
\hline ED visit w/o admit & $10,835(7)$ & $25(7)$ & 0.690 \\
\hline Approach converted & $252(0.16)$ & $5(1.49)$ & $<0.001$ \\
\hline \multicolumn{4}{|l|}{ Aggregate complications, $n(\%)$} \\
\hline Bleeding & $687(0.44)$ & $1(0.3)$ & 0.670 \\
\hline Leak & $747(0.47)$ & $6(1.79)$ & $<0.001$ \\
\hline Cardiovascular & $160(0.1)$ & $2(0.6)$ & 0.005 \\
\hline Pulmonary & $724(0.46)$ & $2(0.6)$ & 0.710 \\
\hline Renal & $209(0.13)$ & $6(1.79)$ & $<0.001$ \\
\hline VTE & $822(0.52)$ & $5(1.49)$ & 0.014 \\
\hline SSI & $1103(0.7)$ & $10(2.98)$ & $<0.001$ \\
\hline Other infection & $1195(0.76)$ & $4(1.19)$ & 0.360 \\
\hline
\end{tabular}

TXP history of solid organ transplantation, LOS post-operative length of stay, CPR cardio-pulmonary resuscitation, DVT deep vein thrombosis, SSI surgical site infection, $C$. diff Clostridium difficile, UTI urinary tract infection, VTE venous thromboembolism

*Median (IQR), interquartile range 
cantly higher in the transplant cohort. Median operative time and post-operative length of stay were significantly longer in the transplant cohort $(p<0.05)$. All 30-day adverse outcomes were higher in the transplant cohort, including significantly higher rates of readmission $(p<0.001)$, intervention $(p=$ $0.039)$, and unplanned ICU admission $(p<0.001)$. While bleeding was similar between the two cohorts, aggregate leak $(p=0.0005)$ and VTE $(p=0.014)$, as well as aggregate cardiovascular, renal, and infectious complications, were significantly higher in the unmatched transplant cohort (Table 2).

\section{Outcomes Following Multivariate Logistic Regression Analysis}

While other variables (history of VTE, chronic steroid use, myocardial infarction, male gender, age, and BMI) conferred a higher mortality risk (Appendix 2 Table 7), we observe that prior solid organ transplantation did not confer a significant overall mortality $(p=0.55)$ or bariatric-related mortality ( $p=$ 0.99 ) risk (Table 3). Even though prior organ transplantation did not confer a mortality difference, it was associated with significantly higher overall morbidity (OR 1.60, $p=0.008)$ and morbidity related to bariatric surgery (OR 1.78, $p=$ $0.004)$. Prior organ transplantation also independently impacted readmission (OR 1.90, $p<0.001$ ), unplanned ICU admission (OR 2.24, $p=0.018$ ), aggregate leak (OR 3.47, $p=$ 0.003 ), and aggregate surgical site infection (OR 3.32, $p<0.001$ ) (Table 3). Other variables impacting bariatricrelated morbidity are shown in Appendix 2 Table 7.

\section{Outcomes Following Matching}

One-to-five propensity matching compared 285 metabolic and bariatric surgery cases with prior solid organ transplantation to 1425 cases without. Cases and controls were statistically similar (Appendix 3 Table 8), except for a higher rate of chronic obstructive lung disease in the cohort without transplantation. Outcomes following propensity matched analysis are detailed in Table 4. Similar to the unmatched cohort analysis, there was no mortality difference between these matched cohorts, but a higher rate of overall morbidity $(10 \%$ vs. $6 \%, p=0.02)$ and bariatric-related morbidity ( $7 \%$ vs. $4 \%, p=0.05)$ in the transplant cohort. While leak rate was three-fold higher in the transplant cohort, the difference was not significant $(p=$ 0.05 ) in this matched analysis.

Case-controlled matching compared 182 cases with 910 equally matched controls (Appendix 4 Table 9). Outcomes are shown in Table 5. Similar to unmatched and propensity matched analyses, operative duration $(p<0.0001)$ and hospital length of stay $(p=0.03)$ remained significantly longer in the transplant cohort after case-control matching. There was no mortality difference. Unlike our unmatched and propensity matched analyses, there was no differences in overall and bariatric-related morbidity after case-control matching. Rates of transfusion requirement ( $2 \%$ vs. $0.22 \%, p=0.009$ ), progressive renal failure $(0.55 \%$ vs. $0 \%, p=0.025)$, and aggregate anastomotic or staple line leak $(2.2 \%$ vs. $0.55 \%, p=$ 0.025 ) remained significantly higher in the transplant cohort, similar to unmatched and propensity matched analyses. All other outcome measures were similar in MBS patients with and without a history of a prior solid organ transplantation (Table 5).

\section{Discussion}

Given the potential for poorer outcomes in obese solid organ transplantation patients, there is significant interest in identifying optimal modalities to achieve significant and durable weight loss, including metabolic and bariatric surgery. The literature regarding the safety of MBS in patients with organ transplantation continues to evolve. Current literature
Table 3 Impact of prior transplantation on bariatric outcomes: multivariate regression analysis

\begin{tabular}{llll}
\hline History of transplant & Odds ratio & $95 \%$ confidence interval & $p$ value \\
\hline Overall mortality & 1.86 & $0.24-14.29$ & 0.550 \\
Bariatric-related mortality & $<0.001$ & $0.001-1000$ & 0.990 \\
Overall morbidity & 1.60 & $1.13-2.28$ & 0.008 \\
Bariatric-related morbidity & 1.78 & $1.12-2.64$ & 0.004 \\
Readmission & 1.90 & $1.30-2.78$ & $<0.001$ \\
Bariatric-related readmission & 2.20 & $1.46-3.34$ & $<0.001$ \\
ICU admission & 2.24 & $1.15-4.37$ & 0.018 \\
Aggregate leak & 3.47 & $1.54-7.87$ & 0.003 \\
Aggregate bleeding & 0.42 & $0.06-3.01$ & 0.390 \\
Aggregate VTE & 2.42 & $0.99-5.92$ & 0.050 \\
Aggregate SSI & 3.32 & $1.72-6.41$ & $<0.001$ \\
\hline
\end{tabular}

ICU intensive care unit, VTE venous thromboembolism, SSI surgical site infection 
demonstrates that MBS is overall safe in transplant patients, but is limited to single-center experiences with small sample sizes $[1,2,12]$.

Utilizing the 2017 MBSAQIP, we show that MBS in TXP patients with prior solid organ transplantation is overall safe, with an associated low mortality. However, there is an increased rate of overall morbidity and bariatric-related morbidity compared with the general bariatric population. For both propensity and case-control matched analyses, operative duration, postoperative length of stay, and progressive renal failure remained

Table 4 Outcomes following 1:5 propensity score matching

\begin{tabular}{|c|c|c|c|}
\hline & $\begin{array}{l}(-) \text { TXP } \\
{[n=1425]}\end{array}$ & $\begin{array}{l}(+) \text { TXP } \\
{[n=285]}\end{array}$ & $p$ value \\
\hline Operative time, $(\mathrm{min})^{*}$ & $76(54-108)$ & $100(67-136)$ & $<0.001$ \\
\hline Hospital LOS (days)* & $2(1-2)$ & $2(1-2)$ & $<0.001$ \\
\hline \multicolumn{4}{|c|}{ 30-day outcomes, and perioperative complications $n(\%)$} \\
\hline Mortality & $2(0.14)$ & $1(0.35)$ & 0.438 \\
\hline Bariatric-related mortality & $1(0.7)$ & $1(0.35)$ & 0.387 \\
\hline Overall morbidity & $87(6)$ & $28(10)$ & $<0.022$ \\
\hline Bariatric-related morbidity & $57(4)$ & $19(7)$ & 0.046 \\
\hline Reoperation & $26(2)$ & $6(2)$ & 0.750 \\
\hline Reoperation related & $22(2)$ & $6(2)$ & 0.495 \\
\hline Readmission & $65(5)$ & $23(8)$ & 0.014 \\
\hline Readmission related & $42(3)$ & $16(6)$ & 0.023 \\
\hline Post-op intervention & $18(1)$ & $6(2)$ & 0.270 \\
\hline Post-op intervention, related & $14(1)$ & $6(2)$ & 0.108 \\
\hline ICU admission & $13(1)$ & $7(2)$ & 0.027 \\
\hline Transfusion & $13(1)$ & $6(2)$ & 0.079 \\
\hline Acute renal failure & $2(0.07)$ & $1(0.35)$ & 0.206 \\
\hline Progressive renal failure & $1(0.07)$ & $1(0.35)$ & 0.002 \\
\hline CPR & $1(0.07)$ & $0(0)$ & 0.655 \\
\hline Stroke & $4(0.28)$ & $0(0)$ & 0.371 \\
\hline Myocardial infarction & $3(0.21)$ & $1(0.35)$ & 0.654 \\
\hline DVT requiring therapy & $7(0.49)$ & $3(1.05)$ & 0.257 \\
\hline Pulmonary embolism & $2(0.14)$ & $1(0.35)$ & 0.438 \\
\hline Pneumonia & $4(0.28)$ & $2(0.7)$ & 0.273 \\
\hline Reintubation & $2(0.14)$ & $0(0)$ & 0.527 \\
\hline Superficial SSI & $7(0.49)$ & $2(0.7)$ & 0.654 \\
\hline Deep incisional SSI & $2(0.14)$ & $1(0.35)$ & 0.438 \\
\hline Organ space SSI & $8(0.56)$ & $2(0.7)$ & 0.740 \\
\hline Post-operative sepsis & $3(0.21)$ & $0(0)$ & 0.438 \\
\hline Post-operative septic shock & $1(0.07)$ & $0(0)$ & 0.655 \\
\hline Post-operative UTI & $8(0.56)$ & $1(0.35)$ & 0.654 \\
\hline C. diff & $4(0.28)$ & $1(0.35)$ & 0.841 \\
\hline Incisional hernia & $2(0.14)$ & $1(0.35)$ & 0.438 \\
\hline ED visit w/o admit & $108(8)$ & $20(7)$ & 0.742 \\
\hline Approach converted & $4(0.28)$ & $3(1.05)$ & 0.062 \\
\hline \multicolumn{4}{|l|}{ Aggregate complications, $n(\%)$} \\
\hline Bleeding & $7(0.49)$ & $1(0.35)$ & 0.751 \\
\hline Leak & $9(0.63)$ & $5(1.75)$ & 0.055 \\
\hline Cardiovascular & $9(0.63)$ & $2(0.7)$ & 0.892 \\
\hline Pulmonary & $11(0.77)$ & $2(0.7)$ & 0.901 \\
\hline Renal & $2(0.14)$ & $4(1.4)$ & 0.001 \\
\hline VTE & $11(0.77)$ & $4(1.4)$ & 0.297 \\
\hline SSI & $11(0.77)$ & $7(2.46)$ & 0.001 \\
\hline Other infection & $20(1.4)$ & $4(1.4)$ & 0.999 \\
\hline
\end{tabular}

TXP history of solid organ transplantation, LOS post-operative length of stay, $C P R$ cardio-pulmonary resuscitation, $D V T$ deep vein thrombosis, SSI surgical site infection, $C$. diff Clostridium difficile, UTI urinary tract infection, VTE venous thromboembolism

*Median interquartile range significantly longer and higher in transplant patients. Some outcome differences were noted between our propensity and casecontrol matched cohorts. While a higher morbidity was noted in the transplant cohort after propensity matched analysis, it did not persist after case-control matched analysis. This was similarly noted for readmission, unplanned ICU admission, aggregate renal complications, and surgical site infection. Across analyses, leak rate remained higher in the transplant cohort. In comparison with propensity matching, case-control matching is often

Table 5 Outcomes following 1:5 case-control matched analysis

\begin{tabular}{|c|c|c|c|}
\hline & $\begin{array}{l}(-) \text { TXP } \\
{[n=910]}\end{array}$ & $\begin{array}{l}(+) \text { TXP } \\
{[n=182]}\end{array}$ & $p$ value \\
\hline Operative time, $(\mathrm{min})^{*}$ & $76(53-108)$ & $104(67-136)$ & $<0.001$ \\
\hline Hospital LOS (days)* & $1(1-2)$ & $2(1-2)$ & 0.030 \\
\hline \multicolumn{4}{|c|}{ 30-day outcomes, and perioperative complications $n(\%)$} \\
\hline Mortality & $0(0)$ & $0(0)$ & 1.000 \\
\hline Bariatric-related mortality & $0(0)$ & $0(0)$ & 1.000 \\
\hline Overall morbidity & $66(7)$ & $14(8)$ & 0.830 \\
\hline Bariatric-related morbidity & $46(5)$ & $9(5)$ & 0.951 \\
\hline Reoperation & $13(1)$ & $4(2)$ & 0.444 \\
\hline Reoperation related & $8(1)$ & $4(2)$ & 0.119 \\
\hline Readmission & $46(5)$ & $12(7)$ & 0.398 \\
\hline Readmission related & $35(4)$ & $8(4)$ & 0.728 \\
\hline Post-op intervention & $14(2)$ & $4(2)$ & 0.524 \\
\hline Post-op intervention, related & $13(1)$ & $4(2)$ & 0.444 \\
\hline ICU admission & $9(1)$ & $4(2)$ & 0.170 \\
\hline Follow-up & $870(96)$ & $171(94)$ & 0.336 \\
\hline Transfusion & $2(0.22)$ & $3(2)$ & 0.009 \\
\hline Acute renal failure & $3(0.33)$ & $0(0)$ & 0.438 \\
\hline Progressive renal failure & $0(0)$ & $1(0.55)$ & 0.025 \\
\hline $\mathrm{CPR}$ & $0(0)$ & $0(0)$ & 1.000 \\
\hline Stroke & $1(0.11)$ & $0(0)$ & 0.655 \\
\hline Myocardial infarction & $0(0)$ & $0(0)$ & 1.000 \\
\hline DVT requiring therapy & $8(1)$ & $3(2)$ & 0.343 \\
\hline Pulmonary embolism & $2(0.22)$ & $1(0.55)$ & 0.438 \\
\hline Pneumonia & $2(0.22)$ & $0(0)$ & 0.527 \\
\hline Reintubation & $1(0.11)$ & $0(0)$ & 0.655 \\
\hline Superficial SSI & $2(0.22)$ & $1(0.55)$ & 0.438 \\
\hline Deep incisional SSI & $2(0.22)$ & $0(0)$ & 0.527 \\
\hline Organ space SSI & $4(0.44)$ & $1(0.55)$ & 0.841 \\
\hline Post-operative sepsis & $0(0)$ & $0(0)$ & 1.000 \\
\hline Post-operative septic shock & $2(0.22)$ & $0(0)$ & 0.527 \\
\hline Post-operative UTI & $5(0.55)$ & $0(0)$ & 0.316 \\
\hline C. diff & $4(0.44)$ & $0(0)$ & 0.370 \\
\hline Incisional hernia & $0(0)$ & $0(0)$ & 1.000 \\
\hline ED visit w/o admit & $68(7)$ & $14(8)$ & 1.000 \\
\hline Approach converted & $2(0.22)$ & $0(0)$ & 0.527 \\
\hline \multicolumn{4}{|l|}{ Aggregate complications, $n(\%)$} \\
\hline Bleeding & $6(1)$ & $0(0)$ & 0.272 \\
\hline Leak & $5(0.55)$ & $4(2.2)$ & 0.025 \\
\hline Cardiovascular & $2(0.22)$ & $0(0)$ & 0.527 \\
\hline Pulmonary & $4(0.44)$ & $0(0)$ & 0.370 \\
\hline Renal & $3(0.33)$ & $1(0.55)$ & 0.654 \\
\hline VTE & $9(1)$ & $4(2.2)$ & 0.170 \\
\hline SSI & $6(0.66)$ & $3(1.65)$ & 0.178 \\
\hline Other infection & $12(1.32)$ & $1(0.55)$ & 0.382 \\
\hline
\end{tabular}

$T X P$ history of solid organ transplantation, LOS post-operative length of stay, $C P R$ cardio-pulmonary resuscitation, $D V T$ deep vein thrombosis, SSI surgical site infection, $C$. diff Clostridium difficile, UTI urinary tract infection, VTE venous thromboembolism

*Median interquartile range 
associated with smaller cohorts that are more tightly matched. This was the case in our analyses, and may have accounted for some of the outcome differences noted between our cohort matching techniques.

Transplantation provides a cure for end stage organ failure, but comes with lifelong immunosuppression. This may account for the increased morbidity in the TXP cohort. In our unmatched analysis, transplant patients were more likely to be on chronic steroids and have preoperative renal insufficiency. Previous matched analyses of the 2015-2016 MBSAQIP database have demonstrated that chronic kidney disease (CKD) and corticosteroid to be independent predictors of morbidity following MBS $[15,16]$. Patients with CKD were more likely to have increased total morbidity, infectious complications, and hospital length of stay [15]. Interestingly, corticosteroid use was an increased risk factor for anastomotic leak (two- to three-fold) but without an increased risk for overall morbidity [16]. The increased leak rate conferred by steroids is prevalent in other surgical disciplines and throughout the literature. Although we control for both of these variables in our propensity and case-control matching techniques, these factors may still contribute to the increase leak rate and morbidity in the TXP cohort. (Reviewer \#1, Comment \#1)

Studies on post-transplantation bariatric surgery are limited, with varied outcomes [1, 12-14, 17]. Khoarki et al. reported their experience with 10 patients undergoing sleeve gastrectomy after liver, kidney, or heart transplant. Mortality and morbidity were $0 \%$ and $20 \%$, respectively. In addition to significant weight loss and resolution of obesity-related conditions, they reported increased graft preservation in liver transplants, improved ejection fraction in heart transplants, and increased estimated glomerular filtration rate in renal transplants [1]. In a case-control matched analysis, Cohen et al. found that post-transplantation bariatric surgery was protective for allograft failure (HR 0.85) and mortality (HR 0.80) [14]. In another single-center small case series, Elli et al. compared outcomes between 10 post-transplant (kidney, liver, or pancreas) and 490 non-transplant LSG patients. Allograft function at 1 year was excellent with $100 \%$ followup in the transplant cohort, and there was no reported mortality or morbidity [12]. Transplant specific analyses show that bariatric surgery was also safe after renal [17] and liver transplantation [13], with low morbidity and mortality. Our study corroborates these findings with no difference in mortality; however we found that prior solid organ transplantation increases the risk of 30-day morbidity and anastomotic leak in MBS patients.

While the published literature suggests that bariatric surgery in transplant patients has an acceptable safety profile, larger cohorts are needed to validate reported outcomes. Even though our study cannot draw conclusions about long-term outcomes (past 30 days), our study is the largest matched cohort study reporting on bariatric surgery outcomes post-transplantation. Similar to published literature, we also found that prior solid organ transplantation did not confer a significantly higher overall or bariatric-related mortality risk, compared with the general bariatric population. However, some post-operative complications remained significantly higher in the transplant cohort (transfusion requirement, renal failure, and leak) after adjusting for potential confounding variables.

Our study has several limitations. This is retrospective analysis of a clinical database that is prone to the inherent biases of such analysis. While the largest reported study on this topic, the overall transplant cohort was small and outcomes are limited to 30 days post-operatively. A sample of cases was excluded that may have impacted our outcomes. We were unable to stratify our transplant cohort by the type of solid organ transplantation performed as this variable is not available in the database. Also unavailable was information on non-solid organ transplant patients; therefore, our findings may not be generalizable to all transplant patients. Due to the small sample sizes, we were unable to stratify our analyses by bariatric procedure type (sleeve vs. gastric bypass) and surgical approach (robotic-assisted vs. conventional laparoscopic). These are potential confounders that may have impacted our findings. To limit procedure-type and surgical approach as potential confounders, these variables were equally matched in both our propensity and case-control matched analyses. Additionally, we lack the timeframe between organ transplantation and metabolic and bariatric surgery, which may impact intraoperative findings, operative course, and ultimately outcome. Finally, information regarding specific immunosuppression regimens for the transplant cohort was not available, and may have also biases biased our findings.

\section{Conclusions}

Despite the recognized limitations in this matched cohort study of the 2017 MBSAQIP database, we found that metabolic and bariatric surgery is overall safe in carefully selected solid organ transplantation patients compared with the general bariatric patient population, with no significant difference in overall and bariatric-related mortality. However, some complications including anastomotic leak remain higher in prior solid organ transplant patients undergoing metabolic and bariatric surgery. Further studies are needed to determine the optimal timing of metabolic and bariatric surgery in this complex patient cohort.

\section{Compliance with Ethical Standards}

Conflict of Interest The authors declare that they have no conflict of interest.

Ethical Statement All procedures performed in studies involving human participants were in accordance with the ethical standards of the institution and/or national research committee and with the 1964 Helsinki declaration and its later amendments or comparable ethical standards. For this study utilizing de-identified data from a national database, formal consent is not required. 
Consent Statement Inform consent was not required as the study used de-identified data from a nationally available database.
Appendix 1. Composite complication methodology

Table 6 Methodology of aggregate complications. For each aggregate complication, composite variables are outlined

\begin{tabular}{|c|c|}
\hline Aggregate variable & Composite variables \\
\hline \multirow[t]{5}{*}{ Leak } & Reoperation with suspected reason: leak \\
\hline & Readmission with suspected reason: leak \\
\hline & Intervention with suspected reason: leak \\
\hline & Drain present over 30 days \\
\hline & Complication: organ space SSI \\
\hline \multirow[t]{3}{*}{ Bleeding } & Reoperation with suspected reason: bleeding \\
\hline & Readmission with suspected reason: bleeding \\
\hline & Intervention with suspected reason: bleeding \\
\hline \multirow[t]{5}{*}{ Cardiac/CVA } & Reoperation with suspected reason: cardiac NOS, CVA, or MI \\
\hline & Readmission with suspected reason: cardiac NOS, CVA, or MI \\
\hline & Intervention with suspected reason: cardiac NOS, CVA, or MI \\
\hline & Complication of CVA \\
\hline & Complication of MI \\
\hline \multirow[t]{6}{*}{ Pulmonary } & Reoperation with suspected reason: shortness of breath, pneumonia, or other respiratory failure \\
\hline & Readmission with suspected reason: shortness of breath, pneumonia, or other respiratory failure \\
\hline & Intervention with suspected reason: shortness of breath, pneumonia, or Other respiratory failure \\
\hline & Complication: on ventilator $>48 \mathrm{~h}$ \\
\hline & Complication: unplanned intubation \\
\hline & Complication: pneumonia \\
\hline \multirow[t]{5}{*}{ Renal } & Reoperation with suspected reason: renal insufficiency \\
\hline & Readmission with suspected reason: renal insufficiency \\
\hline & Intervention with suspected reason: renal insufficiency \\
\hline & Complication: progressive renal insufficiency \\
\hline & Complication: acute renal failure \\
\hline \multirow[t]{6}{*}{ DVT or PE } & Reoperation with suspected reason: vein thrombosis requiring therapy or pulmonary embolism \\
\hline & Readmission with suspected reason: vein thrombosis requiring therapy or pulmonary embolism \\
\hline & Intervention with suspected reason: vein thrombosis requiring therapy or pulmonary embolism \\
\hline & Complication: vein thrombosis requiring therapy \\
\hline & Complication: pulmonary embolism \\
\hline & Complication: anticoagulation initiated of presumed/confirmed vein thrombosis/PE \\
\hline \multirow[t]{5}{*}{ Wound infection } & Reoperation with suspected reason: wound infection or other abdominal sepsis \\
\hline & Readmission with suspected reason: wound infection or other abdominal sepsis \\
\hline & Intervention with suspected reason: wound infection or other abdominal sepsis \\
\hline & Complication: Post-op superficial incisional SSI occurrence \\
\hline & Complication: Post-op deep incisional SSI occurrence \\
\hline \multirow[t]{7}{*}{ Other infection } & Reoperation with suspected reason: infection/fever \\
\hline & Readmission with suspected reason: infection/fever, \\
\hline & Intervention with suspected reason: infection/fever \\
\hline & Complication: post-op sepsis occurrence \\
\hline & Complication: post-op septic shock occurrence \\
\hline & Complication: post-op pneumonia occurrence \\
\hline & Complication: post-op urinary tract infection occurrence \\
\hline \multirow[t]{5}{*}{ Overall morbidity } & Mortality within 30 days \\
\hline & Need for intervention within 30 days \\
\hline & Need for readmission within 30 days \\
\hline & Need for reoperation within 30 days \\
\hline & Unplanned ICU transfer within 30 days \\
\hline \multirow[t]{2}{*}{ Aggregate-related reoperation } & Any reoperation designated as related to metabolic/bariatric by variable \\
\hline & REOP_RELATED_BAR1. To REOP_RELATED_BAR.13 \\
\hline \multirow[t]{2}{*}{ Aggregate-related readmission } & Any readmission designated as related to metabolic/bariatric by variable \\
\hline & READ_RELATED_BAR1. To READ_RELATED_BAR.11 \\
\hline \multirow[t]{2}{*}{ Aggregate-related intervention } & Any intervention designated as related to metabolic/bariatric by variable \\
\hline & INVT_RELATED_BAR1. To INTV_RELATED_BAR.5 \\
\hline \multirow[t]{4}{*}{ Bariatric surgery-related morbidity } & Death related to bariatric surgery \\
\hline & Aggregate reoperation related to metabolic/bariatric surgery \\
\hline & Aggregate readmission related to metabolic/bariatric surgery \\
\hline & Aggregate intervention related to metabolic/bariatric surgery \\
\hline
\end{tabular}




\section{Appendix 2. Independent predictors \\ of mortality and morbidity following sleeve and gastric bypass}

Table 7 Independent predictors of mortality and morbidity following sleeve and gastric bypass following multivariate regression analysis

\begin{tabular}{|c|c|c|c|}
\hline Overall mortality & Odds ratio & $95 \%$ confidence interval & $p$ value \\
\hline VTE & 2.53 & $1.30-4.95$ & 0.006 \\
\hline Chronic steroids & 2.39 & $1.09-5.26$ & 0.030 \\
\hline Myocardial infarction & 2.34 & $1.15-4.78$ & 0.020 \\
\hline Male sex & 2.24 & $1.47-2.99$ & $<0.001$ \\
\hline Anticoagulation for VTE & 2.12 & $1.22-3.68$ & 0.007 \\
\hline Age & 1.05 & $1.04-1.07$ & $<0.001$ \\
\hline BMI & 1.05 & $1.04-1.07$ & $<0.001$ \\
\hline Bariatric-related mortality & Odds ratio & $95 \%$ confidence interval & $p$ value \\
\hline BMI & 1.04 & $1.00-1.09$ & 0.03 \\
\hline Overall morbidity & Odds ratio & $95 \%$ confidence interval & $p$ value \\
\hline History of IVC filter & 1.74 & $1.41-2.15$ & $<0.001$ \\
\hline History of dialysis & 1.70 & $1.28-2.28$ & $<0.001$ \\
\hline History of chronic steroids & 1.55 & $1.35-1.78$ & $<0.001$ \\
\hline History of DVT & 1.53 & $1.32-1.77$ & $<0.001$ \\
\hline History of anticoagulation & 1.46 & $1.30-1.64$ & $<0.001$ \\
\hline History of PE & 1.42 & $1.20-1.67$ & $<0.001$ \\
\hline History of MI & 1.42 & $1.20-1.67$ & $<0.001$ \\
\hline History of cardiac disease & 1.32 & $1.10-1.58$ & 0.003 \\
\hline History of renal insufficiency & 1.26 & $1.00-1.59$ & 0.045 \\
\hline $\mathrm{ASA}>3$ & 1.15 & $1.07-1.22$ & $<0.001$ \\
\hline History of smoking & 1.13 & $1.04-1.22$ & 0.004 \\
\hline History of hyperlipidemia & 1.12 & $1.06-1.20$ & 0.002 \\
\hline History of hypertension & 1.10 & $1.04-1.16$ & 0.001 \\
\hline BMI & 1.01 & $1.00-1.01$ & $<0.00$ \\
\hline Age & 1.00 & $1.00-1.01$ & 0.031 \\
\hline Laparoscopic (vs. robotic) & 0.90 & $0.84-0.98$ & 0.017 \\
\hline Male sex & 0.88 & $0.83-0.94$ & $<0.001$ \\
\hline White (vs. Black) & 0.77 & $0.73-0.82$ & $<0.001$ \\
\hline Bariatric-related morbidity & Odds ratio & $95 \%$ confidence interval & $p$ value \\
\hline History of TXP & 1.78 & $1.12-2.64$ & 0.004 \\
\hline History of DVT & 1.69 & $1.42-2.00$ & $<0.001$ \\
\hline History of IVC filter & 1.55 & $1.21-2.00$ & $<0.001$ \\
\hline History of dialysis & 1.54 & $1.10-2.15$ & 0.012 \\
\hline History of PE & 1.53 & $1.27-1.95$ & $<0.001$ \\
\hline History of MI & 1.48 & $1.22-1.79$ & $<0.001$ \\
\hline History of diabetes & 1.45 & $1.31-1.60$ & $<0.001$ \\
\hline History of chronic steroids & 1.36 & $1.15-1.60$ & 0.003 \\
\hline History of anticoagulation & 1.23 & $1.06-1.41$ & 0.049 \\
\hline ASA $>3$ & 1.17 & $1.09-1.25$ & $<0.001$ \\
\hline History of hyperlipidemia & 1.16 & $1.09-1.24$ & $<0.001$ \\
\hline History of smoking & 1.14 & $1.04-1.24$ & 0.006 \\
\hline BMI & 1.00 & $1.00-1.01$ & 0.002 \\
\hline Laparoscopic (vs. robotic) & 0.90 & $0.82-0.99$ & 0.267 \\
\hline Male sex & 0.82 & $0.77-0.88$ & $<0.001$ \\
\hline
\end{tabular}

$V T E$ venous thromboembolism, $B M I$ body mass index, $I V C$ inferior vena cava, $D V T$ deep vein thrombosis, $P E$ pulmonary emboli, $M I$ myocardial infarction, ASA American Society of Anesthesiologist, TXP history of solid organ transplantation 


\section{Appendix 3. Patient characteristics after 1:5 \\ propensity score matching}

Table 8 Patient characteristics after propensity score matching

\begin{tabular}{|c|c|c|c|}
\hline & $\begin{array}{l}(-) \text { TXP } \\
{[n=1425]}\end{array}$ & $\begin{array}{l}(+) \text { TXP } \\
{[n=285]}\end{array}$ & $p$ value \\
\hline \multicolumn{4}{|l|}{ Continuous variables, median (IQR) } \\
\hline Age (years) & $48(38-57)$ & $48(39-56)$ & 0.832 \\
\hline BMI closest to surgery $\left(\mathrm{kg} / \mathrm{m}^{2}\right)$ & $43.07(39.4-48.55)$ & $42.57(39.53-46.59)$ & 0.204 \\
\hline \multicolumn{4}{|l|}{ Categorical variables, $n(\%)$} \\
\hline Gender (female) & $1048(74)$ & $207(73)$ & 0.750 \\
\hline Race (White) & $748(52)$ & $154(54)$ & 0.634 \\
\hline Race (Black) & $305(21)$ & $62(22)$ & 0.895 \\
\hline Ethnicity (Hispanic) & $247(17)$ & $45(16)$ & 0.527 \\
\hline ASA class & & & 0.945 \\
\hline$<3$ & $158(11)$ & $32(11)$ & \\
\hline$>3$ & $1267(89)$ & $253(89)$ & \\
\hline Operation type & & & 0.438 \\
\hline Sleeve & $1028(72)$ & $212(74)$ & \\
\hline Gastric bypass & $397(28)$ & $73(26)$ & \\
\hline Surgical approach & & & 0.661 \\
\hline Laparoscopic & 1309 (92) & 264 (93) & \\
\hline Robotic & $116(8)$ & $21(7)$ & \\
\hline \multicolumn{4}{|c|}{ Preoperative disease prevalence, $n(\%)$} \\
\hline History of MI & $28(2)$ & $6(2)$ & 0.877 \\
\hline History of PCI & $65(5)$ & $12(4)$ & 0.794 \\
\hline History cardiac surgery & $56(4)$ & $11(4)$ & 0.956 \\
\hline Hypertension & $922(65)$ & $185(65)$ & 0.946 \\
\hline Hyperlipidemia & $537(38)$ & $102(36)$ & 0.546 \\
\hline Diabetes mellitus & $551(39)$ & $102(36)$ & 0.591 \\
\hline COPD & $58(4)$ & $3(1)$ & 0.012 \\
\hline OSA & $455(32)$ & $90(32)$ & 0.908 \\
\hline Oxygen dependent & $34(2)$ & $2(1)$ & 0.071 \\
\hline Smoker & $70(5)$ & $11(4)$ & 0.445 \\
\hline Renal insufficiency & $108(8)$ & $20(7)$ & 0.742 \\
\hline Dialysis & $71(5)$ & $15(5)$ & 0.843 \\
\hline VTE requiring therapy & $42(3)$ & $9(3)$ & 0.849 \\
\hline History of PE & $43(3)$ & $9(3)$ & 0.900 \\
\hline IVC filter & $20(1)$ & $3(1)$ & 0.639 \\
\hline Anticoagulation & $77(5)$ & $18(6)$ & 0.539 \\
\hline Chronic steroids & $455(32)$ & $93(33)$ & 0.817 \\
\hline Limited ambulation status & $46(3)$ & $6(2)$ & 0.316 \\
\hline Independent functional status & $1393(98)$ & $282(99)$ & 0.194 \\
\hline History of bariatric surgery & $106(7)$ & $20(7)$ & 0.804 \\
\hline
\end{tabular}

$T X P$ history of solid organ transplantation, $I Q R$ interquartile range, $B M I$ body mass index, $k g$ kilogram, ASA American Society of Anesthesiologist, $M I$ myocardial infarction, $P C I$ percutaneous coronary intervention, $C O P D$ chronic obstructive pulmonary disease, $O S A$ obstructive sleep apnea, $P E$ pulmonary emboli, $I V C$ inferior vena cava, VTE venous thromboembolism 


\section{Appendix 4. Patient characteristics after 1:5 \\ case-control matched analysis}

Table 9 Patient characteristics after case-control matching

\begin{tabular}{|c|c|c|c|}
\hline & $\begin{array}{l}(-) \text { TXP } \\
{[n=910]}\end{array}$ & $\begin{array}{l}(+) \text { TXP } \\
{[n=182]}\end{array}$ & $p$ value \\
\hline \multicolumn{4}{|l|}{ Continuous variables, median (IQR) } \\
\hline Age (years) & $45(36-75)$ & $46(38-72)$ & 0.45 \\
\hline BMI closest to surgery $\left(\mathrm{kg} / \mathrm{m}^{2}\right)$ & $43.6(39.6-49)$ & $42.6(39.6-47)$ & 0.42 \\
\hline \multicolumn{4}{|l|}{ Categorical variables, $n(\%)$} \\
\hline Gender (female) & $740(81)$ & $148(81)$ & 1 \\
\hline Race (White) & $515(57)$ & $103(57)$ & 1 \\
\hline Race (Black) & $170(19)$ & $34(19)$ & 1 \\
\hline Ethnicity (Hispanic) & $144(16)$ & $29(16)$ & 0.97 \\
\hline ASA class & & & 1 \\
\hline$<3$ & $125(14)$ & $25(14)$ & \\
\hline$>3$ & $785(86)$ & $157(86)$ & \\
\hline Operation type & & & 1 \\
\hline Sleeve & $640(70)$ & $128(70)$ & \\
\hline Gastric bypass & $270(30)$ & $54(30)$ & \\
\hline Surgical approach & & & 1 \\
\hline Laparoscopic & $833(92)$ & $168(92)$ & \\
\hline Robotic & $77(8)$ & $14(8)$ & \\
\hline \multicolumn{4}{|l|}{ Preoperative disease prevalence } \\
\hline History of MI & $5(0.55)$ & $1(0.55)$ & 1 \\
\hline History of PCI & $0(0)$ & $0(0)$ & 1 \\
\hline History cardiac surgery & $5(0.55)$ & $1(0.55)$ & 1 \\
\hline Hypertension & $490(54)$ & $98(54)$ & 1 \\
\hline Hyperlipidemia & $245(27)$ & $49(27)$ & 1 \\
\hline Diabetes mellitus & $250(27)$ & $50(27)$ & 1 \\
\hline COPD & $25(3)$ & $3(2)$ & 0.39 \\
\hline OSA & $240(26)$ & $48(26)$ & 1 \\
\hline Oxygen dependent & $8(1)$ & $1(1)$ & 1 \\
\hline Smoker & $35(4)$ & $7(4)$ & 1 \\
\hline Renal insufficiency & $0(0)$ & $0(0)$ & 1 \\
\hline Dialysis & $0(0)$ & $0(0)$ & 1 \\
\hline VTE requiring therapy & $0(0)$ & $0(0)$ & 1 \\
\hline History of PE & $5(0.55)$ & $1(0.55)$ & 1 \\
\hline IVC filter & $5(0.55)$ & $1(0.55)$ & 1 \\
\hline Anticoagulation & $5(0.55)$ & $1(0.55)$ & 1 \\
\hline Chronic steroids & $155(17)$ & $31(17)$ & 1 \\
\hline Limited ambulation status & $6(1)$ & $3(2)$ & 0.18 \\
\hline Independent functional status & $898(99)$ & $182(100)$ & 0.12 \\
\hline History of bariatric surgery & $63(7)$ & $10(5)$ & 0.48 \\
\hline
\end{tabular}

TXP history of solid organ transplantation, $I Q R$ interquartile range, $\mathrm{kg}$ kilogram, ASA American Society of Anesthesiologist, $M I$ myocardial infarction, $P C I$ percutaneous coronary intervention, $C O P D$ chronic obstructive pulmonary disease, $O S A$ obstructive sleep apnea, $P E$ pulmonary emboli, IVC inferior vena cava, VTE venous thromboembolism 


\section{References}

1. Khoraki J, Katz MG, Funk LM, et al. Feasibility and outcomes of laparoscopic sleeve gastrectomy after solid organ transplantation. Surg Obes Relat Dis. 2016;12(1):75-83.

2. Kienzl-Wagner K, Weissenbacher A, Gehwolf P, et al. Laparoscopic sleeve gastrectomy: gateway to kidney transplantation. Surg Obes Relat Dis. 2017;13(6):909-15.

3. Leonard J, Heimbach JK, Malinchoc M, et al. The impact of obesity on long-term outcomes in liver transplant recipients-results of the NIDDK liver transplant database. Am J Transplant. 2008;8:667-72.

4. Rezende Anastacio LR, Garcia Ferreira L, Costa Liboredo J, et al. Overweight, obesity and weight gain up to three years after liver transplantation. Nutr Hosp. 2012;27:1351-6.

5. Beckmann S, Natasa I, Drent G, et al. Weight gain, overweight and obesity in solid organ transplanation-a study protocol for a systematic literature review. Syst Rev. 2015;4(1):1-16.

6. Beckmann S, Nikolic N, Denhaerynck K, et al. Evolution of body weight parameters up to 3 years after solid organ transplantation: the prospective Swiss Transplant Cohort Study. Clin Transplant. 2017;31(3)

7. Nicoletto BB, Fonseca NK, Manfro RC, et al. Effects of obesity on kidney transplantation outcomes: a systematic review and metaanalysis. Transplantation. 2014;98:167-76.

8. Sood A, Hakim DN, Hakim NS. Consequence of recipient obesity on postoperative outcomes in a renal transplant: a systematic review and meta-analysis. Exp Clin Transplant. 2016;14(2):121-8.

9. Kim Y, Jung AD, Dhar VK, et al. Laparoscopic sleeve gastrectomy improves renal transplant candidacy and posttransplant outcomes in morbidly obese patients. Am J Transplant. 2018;18(2):410-6.
10. Serrano OK, Sengupta B, Bangdiwala A, et al. Implications of excess weight on kidney donation: long-term consequences of donor nephrectomy in obese donors. Surgery. 2018;164(5):1071-6.

11. Diwan TS, Rice TC, Heimbach JK, et al. Liver transplantation and bariatric surgery: timing and outcomes. Liver Transpl. 2018;24(9): 1280-7.

12. Elli EF, Gonzalez-Heredia R, Sanchez-Johnsen L, et al. Sleeve gastrectomy surgery in obese patients post-organ transplantation. Surg Obes Real Dis. 2016;12(3):528-34.

13. Osseis M, Lazzati A, Salloum C, et al. Sleeve gastrectomy after liver transplantation: feasibility and outcomes. Obes Surg. 2018;28(1):242-8.

14. Cohen JB, Lim MA, Tewksbury CM, et al. Bariatric surgery before and after kidney transplantation: long-term weight loss and allograft outcomes. Surg Obes Relat Dis. 2019;15(6):935-41.

15. Mazzei M, Zhao H, Edwards MA. The impact of chronic kidney disease on bariatric perioperative outcome: a MBSAQIP matched analysis. Surg Obes Relat Dis. 2019;15(12):2075-86.

16. Mazzei M, Zhao H, Edwards MA. Perioperative outcomes of bariatric surgery in the setting of chronic steroid use: an MBSAQIP database analysis. Surg Obes Relat Dis. 2019;15(6):926-34.

17. Gazzetta PG, Bissolati M, Salbene A, et al. Bariatric surgery to target obesity in the renal transplant population: preliminary experience in a single center. Transplant Proc. 2017;49(4):646-9.

Publisher's Note Springer Nature remains neutral with regard to jurisdictional claims in published maps and institutional affiliations. 